\title{
A case of dengue fever presenting with acute urticaria
}

\author{
Mati Chuamanochan, ${ }^{1,2}$ Siri Chiewchanvit, ${ }^{1}$ Napatra Tovanabutra, ${ }^{1}$ Rujira Rujiwetpongstorn, ${ }^{1}$ Kulwadee Laosakul, ${ }^{1}$ Marcus Maurer ${ }^{2}$
}

\begin{abstract}
Background: Acute urticaria (AU) is characterized by the occurrence of spontaneous wheals, angioedema, or both for less than 6 weeks. Viral infection is considered to be one of the common causes of AU, however AU has never been reported in association with dengue infection.
\end{abstract}

Objective: To describe the first case of AU in dengue virus infection.

\section{Methods: Case report}

Results: A 17-year-old healthy male presented with first episode of AU which associated with confirmed dengue virus infection. He responded well with antihistamine treatment and without recurrence at 2-month follow up.

Conclusion: Patients with dengue virus infections can present with AU, possibly from viral-infected mast cell. Future research will have to clarify this association.

Key words: acute urticaria, dengue fever, mast cell, virus infection, wheal

From:

${ }^{1}$ Division of Dermatology, Department of Internal Medicine, Faculty of Medicine, Chiang Mai University, Chiang Mai, Thailand

2 Dermatological Allergology, Department of Dermatology and Allergy, Charité - Universitätsmedizin Berlin, Germany

\section{Introduction}

Urticaria is a characteristic mast cell-driven disease, manifests with wheals and/or angioedema. ${ }^{1}$ Current classification defines acute urticaria $(A U)$ by the duration of disease that is less than 6 weeks. $^{2}$ In adult, the most common causes of AU are infections, follows by drug hypersensitization. ${ }^{3}$ Among infectious etiology, upper respiratory tract infections were mostly reported from previous studies. ${ }^{4-6}$ Our case report demonstrates a case of dengue virus infection presenting with AU. From the literature review, AU has never been described as a dermatologic finding in dengue infection.

\section{Case report}

A 17-year-old healthy male presented with an itchy rash that had begun on the back, before spreading to the entire body, followed within 2 hours by fever and myalgia. When the symptoms persisted for 6 hours, he decided to visit our hospital. The patient denied having any previous rash with this morphology. For the patient himself as well as for his family, the history for allergic diseases and urticaria was negative. Also, our patient was not taking any medication, and he reported

\author{
Corresponding author: \\ Marcus Maurer \\ Charité - Universitätsmedizin Berlin \\ Department of Dermatology and Allergy \\ Charitéplatz 1, 10117 Berlin, Germany \\ E-mail: marcus.maurer@charite.de
}

regular food consumption. On the day of admission, he was febrile with a temperature of $39.7^{\circ} \mathrm{C}$.

Dermatological inspection revealed generalized and partially confluent itchy wheals surrounded by mild reflex erythema over the face, trunk and all extremities (Figure 1) without angioedema. Complete blood count (CBC) showed mild leukocytosis with neutrophil predominance. The dengue nonstructural protein 1 (NS1) antigen test was positive. IgM and IgG antibodies to dengue virus, Orientia tsutsugamushi and leptospira were negative. Dengue fever was the provisional diagnosis.

The patient was admitted and treated with intravenous chlorpheniramine. Individual lesions resolved without changes in pigmentation within 24 hours. After three days in the hospital, fever and rash were completely absent. CBC on day 5 of the illness showed leukopenia with lymphocyte predominance. The patient was discharged without any complications.

At a 2-month follow up visit, the patient reported no recurrence of any rash. Repeated IgM and IgG antibodies to dengue virus were both positive. Dengue fever was the definite diagnosis. 

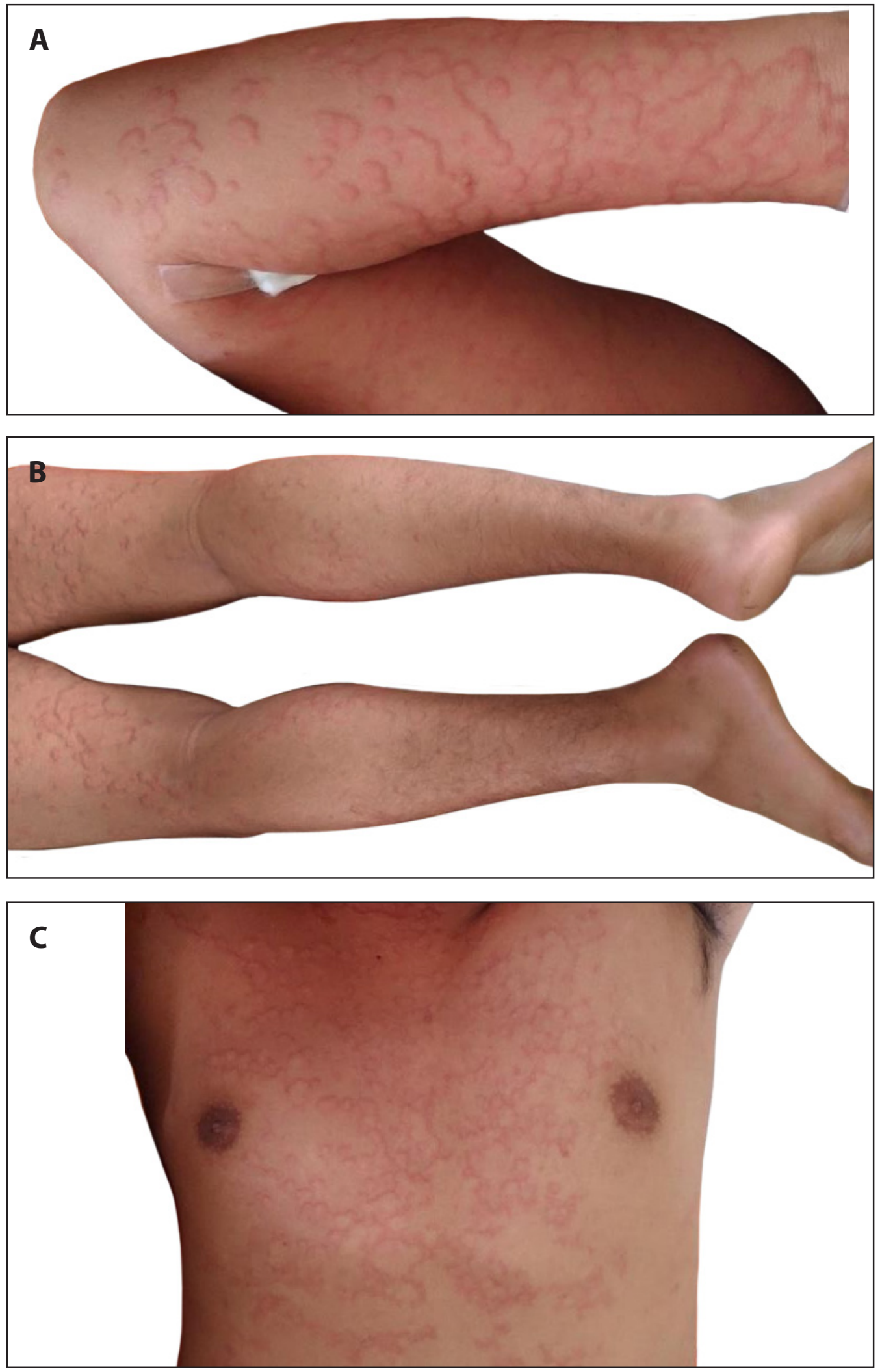

Figure 1. Multiple and partially confluent wheals surrounded by mild reflex erythema on the right ventral forearm (A), legs (B), and chest $(\mathrm{C})$. 


\section{Discussion}

Dengue fever (DF) is a mosquito-borne tropical infection caused by the dengue virus. ${ }^{7}$ Aedes aegypti is a common vector. ${ }^{8}$ Skin rashes are one of the frequent clinical presentations in DF, reported in $47-82 \%$ of cases, and include transient flushing erythema of the face, maculopapular or morbilliform eruptions, and convalescent rashes ("white islands in a sea of red"). ${ }^{9-11}$

Infections are a possible trigger or cause of urticaria. In structured reviews, Wedi et al. ${ }^{12}$ and Imbalzano et al. ${ }^{13}$ demonstrated the association of urticaria with several viruses, namely human immunodeficiency virus; influenza; adenovirus; enterovirus; rotavirus; respiratory syncytial virus; hepatitis A, B and $C$ viruses; herpes simplex virus 1 and 2; human herpesvirus 6; cytomegalovirus; Epstein-Barr virus; parvovirus B19 and norovirus. However, this association is difficult to establish, because rechallenge of the suspected agents is not possible. Several pathomechanisms have been implied to contribute to urticaria due to viral infections, including (i) mast cell degranulation induced by IgM, IgG and IgE against viral antigens, (ii) circulating immune complexes that activate basophils, mast cells and complement, (iii) hyperbilirubinemia resulting in increased vascular permeability (in the case of viral hepatitis) and (iv) proteins secreted from the intestine during viral hepatitis infections inducing the release of pro-inflammatory mediators that augment vascular permeability. ${ }^{13}$

To the best of our knowledge, this is the first report of AU associated with dengue infection. AU was a definite diagnosis as all urticarial lesions resolved within 24 hours and the total duration of urticaria was only 3 days. The close relationship of the onset and resolution of $\mathrm{AU}$ and dengue fever, as well as the lack of other common causes of AU such as the intake of drugs or the consumption of food support a causal link of dengue fever and $\mathrm{AU}$ in our case. However, urticaria may be more common in patients with dengue fever than previously thought. In a recent report, $2 \%$ of a sizeable cohort of patients with DF reportedly had urticaria, and $8 \%$ had an urticaria-like rash. ${ }^{14}$ Future studies in DF will have to clarify the true prevalence of acute and chronic urticaria as well as its subforms, i.e. spontaneous and inducible.

The role of human skin mast cells (MC) in dengue infection may explain this. Recently, the dengue virus has been reported to primarily infect MCs, which is held to contribute to severe dengue manifestations. Dengue virus has also been described to induce MC degranulation and the release of MC products. ${ }^{15-17}$ These released mediators, including histamine, increase vascular permeability, and could account for the wheals in our patient. AU may be an early sign of dengue virus infection. Further studies of the association between dengue infection and urticaria are needed.

\section{Funding Source}

This study was supported by intramural funding.

\section{Conflict of interest}

None

\section{References}

1. Radonjic-Hoesli S, Hofmeier KS, Micaletto S, Schmid-Grendelmeier P, Bircher A, Simon D. Urticaria and Angioedema: an Update on Classification and Pathogenesis. Clin Rev Allergy Immunol. 2018;54:88-101.

2. Zuberbier T, Aberer W, Asero R, Bindslev-Jensen C, Brzoza Z, Canonica GW, et al. The EAACI/GA(2) LEN/EDF/WAO Guideline for the definition, classification, diagnosis, and management of urticaria: the 2013 revision and update. Allergy. 2014;69:868-87.

3. Maurer M, Grabbe J. Urticaria: its history-based diagnosis and etiologically oriented treatment. Dtsch Arztebl Int. 2008;105:458-65; quiz 65-6.

4. Zuberbier T, Ifflander J, Semmler C, Henz BM. Acute urticaria: clinical aspects and therapeutic responsiveness. Acta Derm Venereol. 1996;76: 295-7.

5. Aoki T, Kojima M, Horiko T. Acute urticaria: history and natural course of 50 cases. J Dermatol. 1994;21:73-7.

6. Kulthanan K, Chiawsirikajorn Y, Jiamton S. Acute urticaria: etiologies, clinical course and quality of life. Asian Pac J Allergy Immunol. 2008;26: 1-9.

7. Simmons CP, Farrar JJ, Nguyen v V, Wills B. Dengue. N Engl J Med. 2012;366:1423-32.

8. Cucunawangsih, Lugito NPH. Trends of Dengue Disease Epidemiology. Virology (Auckl). 2017;8:1178122X17695836.

9. Thomas EA, John M, Kanish B. Mucocutaneous manifestations of Dengue fever. Indian J Dermatol. 2010;55:79-85.

10. Huang HW, Tseng HC, Lee CH, Chuang HY, Lin SH. Clinical significance of skin rash in dengue fever: A focus on discomfort, complications, and disease outcome. Asian Pac J Trop Med. 2016;9:713-8.

11. Korman AM, Alikhan A, Kaffenberger BH. Viral exanthems: An update on laboratory testing of the adult patient. J Am Acad Dermatol. 2017;76: 538-50.

12. Wedi B, Raap U, Wieczorek D, Kapp A. Urticaria and infections. Allergy Asthma Clin Immunol. 2009;5:10.

13. Imbalzano E, Casciaro M, Quartuccio S, Minciullo PL, Cascio A, Calapai $\mathrm{G}$, et al. Association between urticaria and virus infections: A systematic review. Allergy Asthma Proc. 2016;37:18-22.

14. Qiu FX, Gubler DJ, Liu JC, Chen QQ. Dengue in China: a clinical review. Bull World Health Organ. 1993;71:349-59.

15. Troupin A, Shirley D, Londono-Renteria B, Watson AM, McHale C, Hall A, et al. A Role for Human Skin Mast Cells in Dengue Virus Infection and Systemic Spread. J Immunol. 2016;197:4382-91.

16. Londono-Renteria B, Marinez-Angarita JC, Troupin A, Colpitts TM. Role of Mast Cells in Dengue Virus Pathogenesis. DNA Cell Biol. 2017;36:423-7.

17. Syenina A, Jagaraj CJ, Aman SA, Sridharan A, St John AL. Dengue vascular leakage is augmented by mast cell degranulation mediated by immunoglobulin Fcgamma receptors. Elife. 2015;4. 